\title{
77. POSSIBLE INFLUENCE OF THE GALACTIC SPIRAL STRUCTURE ON THE LOCAL DISTRIBUTIONS OF STELLAR RESIDUAL VELOCITIES
}

\author{
M. MAYOR \\ Observatoire de Genève, Genève, Switzerland
}

The residual velocity distribution, observed in the solar neighbourhood, exhibits several peculiarities for the stars of low epicyclic energy: vertex deviation, two-stream distribution, etc.

There are mainly two interpretations of these observational facts: direct influence of initial conditions or irregularities of the gravitational potential. Samples of stars (such as the stars closer than $20 \mathrm{pc}$ from the sun, or samples of giant stars) having practically all performed several galactic revolutions do present the aforementioned peculiarities for low epicyclic energy. It seems hard to admit that the initial conditions of these 'old' stars could produce such peculiarities.

We have worked out an explanation of these distribution anomalies as a result of the self-sustained density waves considered by Lin and Shu (1964). We adopted, for the parameters of spiral structure, the values given by Lin et al. (1969), viz.

$$
\Omega_{\mathrm{p}} \approx 12 \mathrm{~km} \mathrm{~s}^{-1} \mathrm{kpc}^{-1} ; \frac{\text { spiral field }}{\text { mean field }} \approx 5 \% ; \quad i \approx 6^{\circ},
$$

where $\Omega_{\mathrm{p}}$ is the pattern angular velocity and $i$ the pitch angle, at the sun's position, of the trailing arm.

The linearized theory of a stellar population's response to the density wave has led to a satisfactory qualitative agreement with the observations about the vertex deviation and its dependence on the velocity dispersion.

However, a detailed calculation of the residual velocity distribution function, perturbed by a spiral field, is out of the range of validity of the linear theory if the stellar populations have small velocity dispersions. In order to compare the calculated and observed loci of equal density in the plane $\left(c_{w}, c_{\theta}\right)$ of residual velocity components, we have obtained the non linear response to the spiral potential.

The Liouville equation for the relative perturbation $\psi$ due to the spiral potential reads symbolically

$$
L_{0}\{\psi\}=L_{1}\left\{Q_{0}\right\}+\left[\psi L_{1}\left\{Q_{0}\right\}-L_{1}\{\psi\}\right]
$$

where $\Psi=\Psi_{0}(1+\psi)$ is the perturbed distribution function and $\Psi_{0}$ the equilibrium distribution, and $Q_{0}=-\ln \Psi_{0} . L_{0}$ and $L_{1}$ are differential operators defined by Lin (1967).

The complete Equation (2), including non linear perturbation terms within the brackets, has been solved by an iterative process.

The response of various stellar populations moving across the density wave defined 
by (1) has been calculated. Locating the sun on the inner edge of a spiral arm, it is possible to reach a satisfactory agreement with the observed distributions; the computed distributions allow for a correct vertex deviation in sign as well as in magnitude; they also reveal a two stream structure.

It therefore seems possible to explain the 'anomalies' of residual velocity distribution for low epicyclic energy in terms of a spiral density wave.

A paper containing the above results has been submitted for publication in Astronomy and Astrophysics.

\section{Acknowledgements}

I wish to express my gratitude to Prof. P. Bouvier and Dr. L. Martinet for many stimulating and useful discussions during this study.

\section{References}

Lin, C. C.: 1967, in Relativity Theory and Astrophysics, 2 Galactic Structure Amer. Math. Soc., Providence, p. 66.

Lin, C. C. and Shu, F. H.: 1964, Astrophys. J. 140, 646.

Lin, C. C., Yuan, C., and Shu, F. H.: 1969, Astrophys. J. 155, 721. 